\title{
Interlinked temporal changes in environmental conditions, chemical characteristics of sediments and macrofaunal assemblages in an estuarine intertidal sandflat (Seto Inland Sea, Japan)
}

\author{
Paolo Magni • Serena Como • Shigeru Montani • \\ Hiroaki Tsutsumi
}

Published online: 16 September 2006

(C) Springer-Verlag 2006

\section{Erratum to: Mar Biol 149(5):1185-1197 DOI 10.1007/s00227-006-0298-0}

At the proof correction stage, the author overlooked a significant error in Table 1. Please find the correct version below.

Table 1 Correlation coefficient $R$ between hydrological variables

\begin{tabular}{|c|c|c|c|c|c|c|}
\hline & Temp & Sal & DO & Chl $a$ & Pheop & POC \\
\hline DO & & $0.68 * *(n=19)$ & & & & \\
\hline $\mathrm{NH}_{4}^{+}-\mathrm{N}$ & $-0.62 * *(n=23)$ & $-0.52 * *(n=27)$ & $-0.56 * *(n=21)$ & & & \\
\hline Pheop & & $-0.62 * *(n=26)$ & & $0.42 *(n=35)$ & & \\
\hline POC & & $-0.61 * *(n=27)$ & & $0.40 *(n=35)$ & $0.99 * * *(n=35)$ & \\
\hline TSM & & $-0.52 * *(n=27)$ & & & $0.95^{* * *}(n=35)$ & $0.96^{* * *}(n=36)$ \\
\hline
\end{tabular}

Only statistically significant correlations are indicated: $* P<0.05, * * P<0.01, * * * P<0.001$; in bold: negative correlations $D O$ dissolved oxygen, Chl a chlorophyll $a$, Pheop pheopigments, POC particulate organic carbon, TSM total suspended matter, $n$ number of samples

The online version of the original article can be found at http://dx.doi.org/10.1007/s00227-006-0298-0.

P. Magni $(\bowtie) \cdot$ S. Como

IMC - International Marine Centre,

Località Sa Mardini, Torregrande,

09072 Oristano, Italy

e-mail: p.magni@imc-it.org

S. Montani

Graduate School of Environmental Science,

Hokkaido University, Kita 13 Nishi 8, Kita-ku,

Sapporo, 060-0813 Hokkaido, Japan

H. Tsutsumi

Faculty of Environmental and Symbiotic Sciences,

Prefectural University of Kumamoto,

3-1-100 Tsukide, 863-8502 Kumamoto, Japan 